\title{
Mass spectrometry and biomarker development
}

\author{
Karin D. Rodland \\ Biological Sciences Division, Pacific Northwest National Laboratory, 902 Battelle Blvd, Richland, WA 99352, USA \\ E-mail: Karin.Rodland@pnl.gov
}

The natural history of disease is usually a story of progression from an early stage in which homeostasis is only mildly perturbed and easily restored, through a series of increasingly involved stages characterized by a steady increase in the extent and impact of pathological change. Eventually the disease process becomes irreversible, and treatment is ameliorative at best. One of the major goals of twenty-first century medicine is the identification of biomarkers for the earliest possible stages of disease involvement so that prompt clinical intervention can limit damage, reverse pathological change, and ideally effect a complete cure.

New developments in the analytical field of mass spectrometry are providing clinicians and translational scientists with ever more powerful tools for the discovery of novel biomarkers. New developments range from significant improvements to the sensitivity and throughput of classic approaches such as 2D-PAGE and protein ELISAs, through application of ancillary technologies such as laser capture microdissection and high density microarray printing, to the development of new instrument platforms providing unique capabilities. The impact of SELDI-MS (Surface Enhanced Laser Desorption and Ionization Mass Spectrometry) on the widespread use of mass spectrometry for biomarker detection is highly significant. SELDIMS provides a relatively sensitive and straightforward approach for the measurement of mass to charge ratios $(\mathrm{m} / \mathrm{z})$ in protein and peptide samples that have been rendered less complex by application to 'protein chips', derivatized surfaces that retain a subset of the protein sample based on chemical properties such as hydrophobicity, positive or negative charge, or recognition by specific affinity reagents. SELDI-MS provides a 'protein profile', a list of peak $\mathrm{m} / \mathrm{z}$ values and a measure of peak intensity. The availability of information about multiple proteins and peptides at a time, without individual identification of each protein, has prompted the development of new statistical algorithms for interpreting and applying these protein profiles to biomarker discovery. Mass spectrometers in general continue to show a steady increase in technical parameters, including resolving power, measured mass accuracy, and dynamic range. Instrument platforms that were once the sole province of sophisticated instrument development labs, like the Fourier Transform Ion Cyclotron Resonance Mass Spectrometers (FTICR-MS) developed at Pacific Northwest National Laboratory, are now available, in a somewhat simplified form, from several commercial vendors. The availability of these highly capable instruments in numerous academic research centers will inevitably lead to an increasing use of the Accurate Mass and Time (AMT) tag approach to protein identification. The advantages of the AMT approach in identifying large numbers of proteins with confidence has recently been demonstrated in the context of the HUPO Plasma Proteome Pilot Project.

The number of disease models that have been approached via proteomic methods is also increasing, as the available instrumentation becomes more robust and accessible. This special issue of Disease Markers includes examples of biomarker discovery efforts directed at breast and prostate cancers, diabetes mellitus, and heart disease. A key requirement for the successful application of mass spectrometric approaches to biomarker discovery is a clear reference standard for 
the 'normal' human proteome, and a better understanding of the sources and extent of 'normal' variability in human plasma proteins. The Human Proteome Organization (HUPO) is undertaking a major collaborative study of human blood samples, comparing serum versus plasma, sample preparation and storage, analytical platform, and bioinformatics tools. This Human Plasma Proteome Project promises to provide a solid common foundation for future efforts at biomarker discovery that cross laboratories and permit data integration and meta-analysis. 


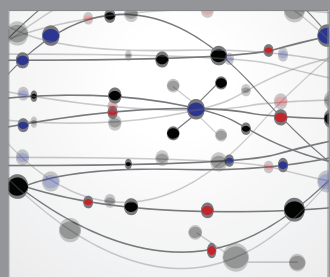

The Scientific World Journal
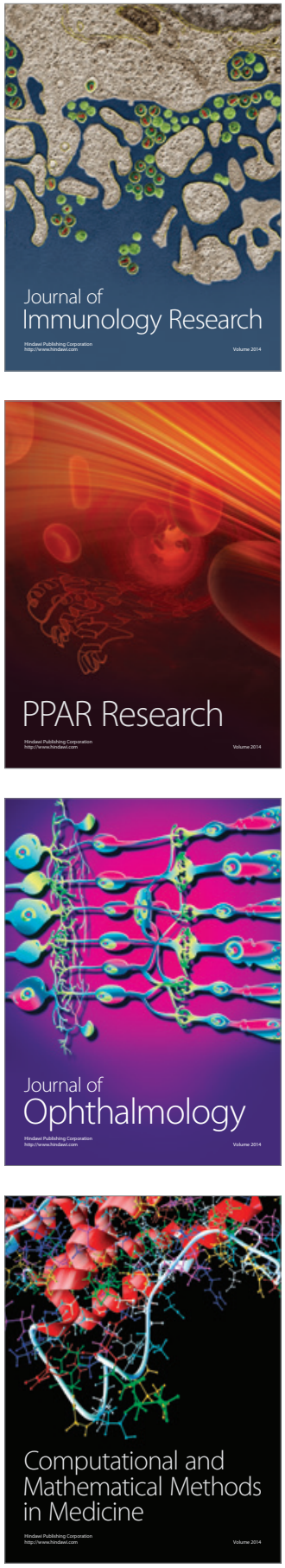

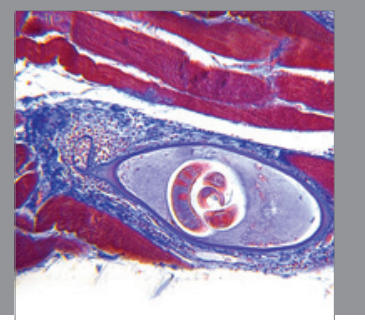

Gastroenterology

Research and Practice
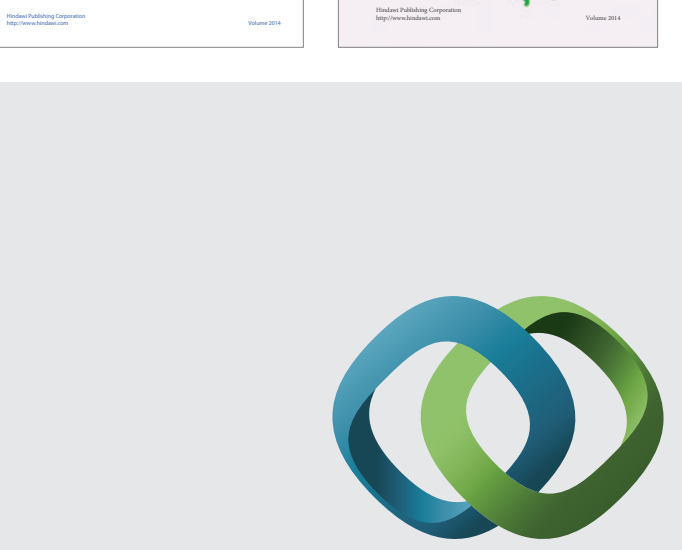

\section{Hindawi}

Submit your manuscripts at

http://www.hindawi.com
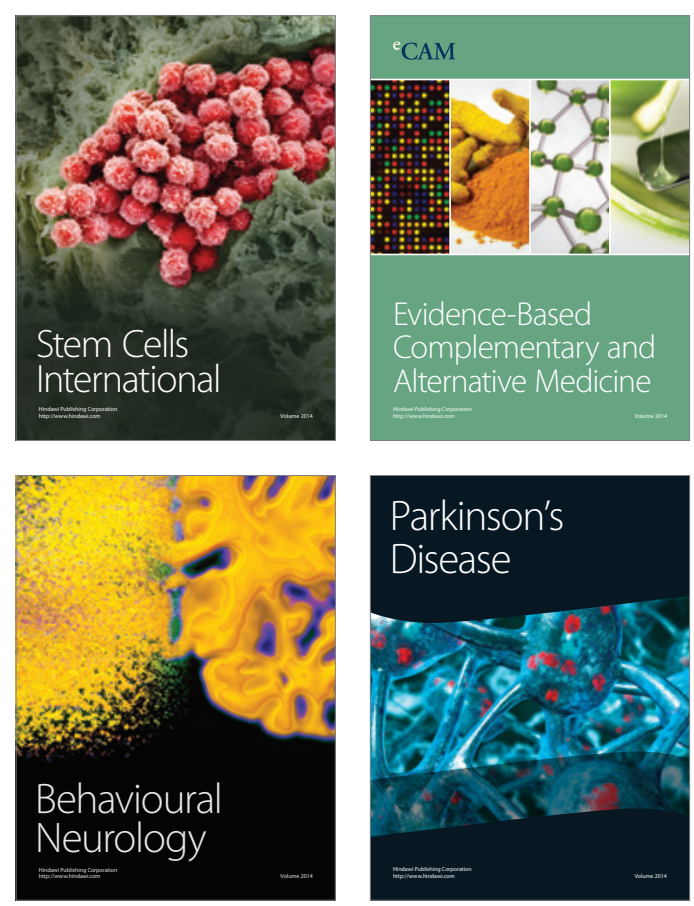

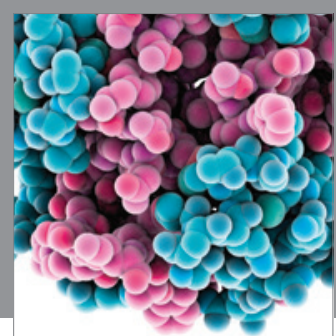

Journal of
Diabetes Research

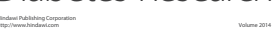

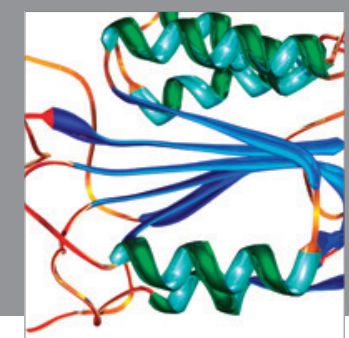

Disease Markers
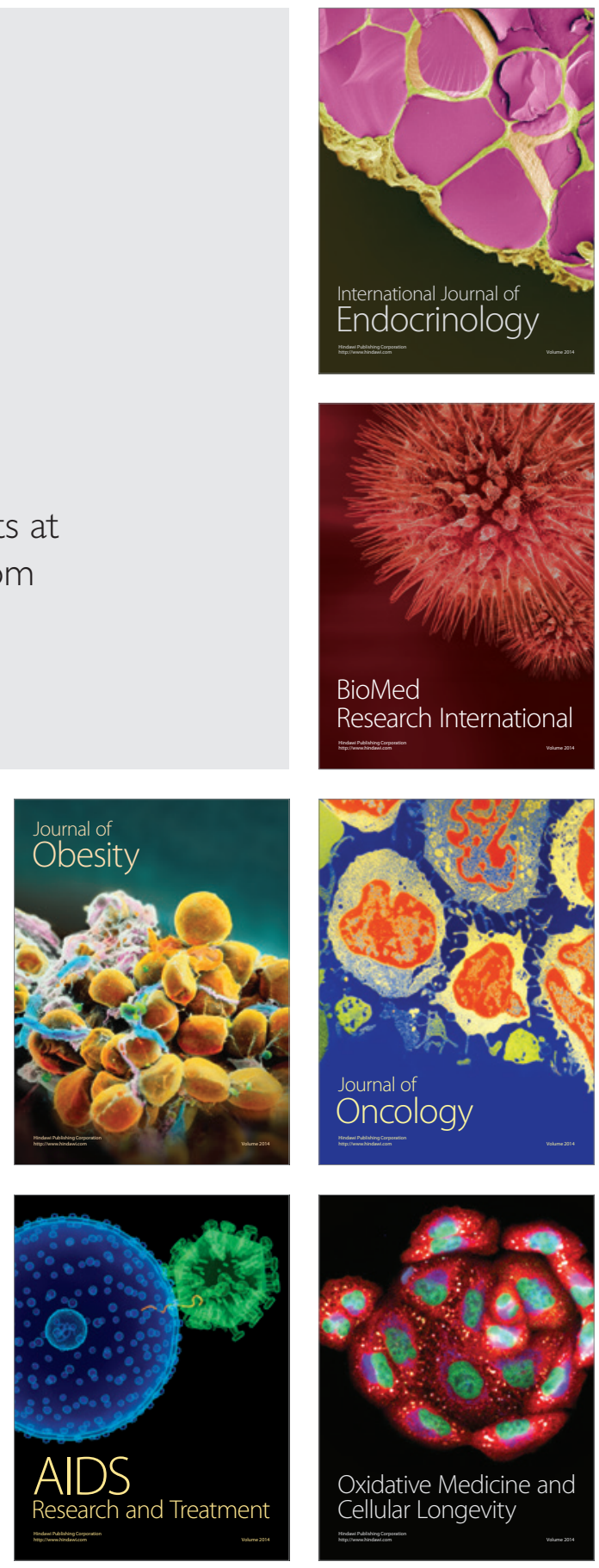